\title{
Mechanisms of CC14 Retention and Slow Release in Model Porous Solids and Sediments
}

\author{
Brent M. Peyton \\ Dana Hall Room 118 \\ Center for Multiphase Environmental Research \\ Washington State University \\ Pullman, WA 99164-2710
}

This work is part of a larger collaborative project of the same title led by Robert Riley at PNNL. Our task goal is to use a state of the art microbalance and well-defined mesoporous silica particles to characterize the effects of pore size distribution on carbon tetrachloride release rate and sequestration. Mesoporous silicon dioxide (SiO2) particles with pore size distribution in the range of 20 to 70 Angstroms were synthesized under acidic conditions using nonionic surfactants as structure directing agents (Zhao et al., 1998). Siliceous particles (primarily silica dioxide) containing the template molecules are precipitated from solution, dried and the template is removed by combustion at $550 \mathrm{C}$. The particles obtained by this procedure were characterized using standard nitrogen adsorption at $77 \mathrm{~K}$. Surface area and pore size distribution were calculated from the data coming from the nitrogen experiments. The particle size distribution was determined by using a centrifugal automatic particle size distribution analyzer, which employs a non-contact measuring method based on liquid-phase sedimentation and the change in particle concentrations on the basis of light transmission. To examine the effects of the pore size on CCl4 sequestration and release, a state-of-the-art microbalance was used to measure real-time desorption profiles of CCl4 released from these mesoporous materials. In addition, a gas chromatograph with flammable ionization detector (FID) and electron capture detector (ECD) was used to close the mass balances and confirm the observations made with the microbalance. Experiments with the microbalance include helium calibration (critical to the measurement of small amounts of CCl4 release), and CCl4 desorption rate experiments, to quantify release rates and residual sequestration of $\mathrm{CCl} 4$ in the mesoporous silica particles. Finally, a fully automated accelerated solvent extraction system was used to determine the actual mass of CT remaining in the samples immediately after finishing the desorption experiments.

\section{Results and Discussion}

The isotherms obtained in the nitrogen experiments for Tween40 particles were of type IVa according to the classification proposed by IUPAC and allowed the calculation of the surface area by employing the Brunauer-Emmett-Teller (BET) method $(611 \mathrm{~m} 2 / \mathrm{g})$. To verify this result, the surface area was also calculated by using alpha-s and t methods which yield larger values than the BET method (727 and 722 $\mathrm{m} 2 / \mathrm{g}$ respectively). The difference between these values is considered to be normal and is related to the pore structure and the proportions of the surface area of mesopores and macropores. The desorption isotherm was used to determine the pore size distribution as suggested by Sing (1998). BJH, Halsey, Broekoff-de Boer and Naono?s methods were used in the calculation of the pore size distribution. The method proposed by Naono (1997) is intended to calculate the PSD of mesoporous materials in the range of 1-5 nm, and is based on a modified Kelvin equation and the consideration of a different film thickness for curve and flat pore surfaces. All the methods used produced curves showing a quite narrow pore size distribution with peak pore diameters centered at 20-23 Angstroms, with exception of the Naono?s model, which showed a peak at 28 Angstroms. In accordance with Naono?s model, the adsorbed thickness in curve pores is larger due to enhanced adsorption, which shifts a PSD calculated using this method to a higher value. The particle size distribution measured by liquid-phase sedimentation showed a peak centered around 20 microns with a median of 12 microns and an average diameter equal to 19 microns. Desorption rate experiments were carried out using Tween 40 particles. In general, approximately 0.9 g of sample (mass calculated from the helium calibration) were put in the microbalance cell and then loaded with CT saturated vapor. Next, the particles were swept at $25 \mathrm{C}$ with helium at a flow rate of $30 \mathrm{ml} / \mathrm{min}$ for the duration of the long-term experiment. During the first $150 \mathrm{~h}$ of sweep, the CT desorption rates calculated from the microbalance (MB) readings decreased gradually and were in very good agreement with those calculated by gas chromatography. After this period of time the MB readings became unstable and the rates of desorption were determined only by GC. After 330 h, the rate of desorption was 3.1x10-7 g CT/(g 
sample $\mathrm{h}$ ) and the mass adsorbed $0.00121 \mathrm{~g} / \mathrm{g}$ sample, which implies that rates were dropping faster than the CT mass was being removed, thus very long periods of time would be required completely remove all the CT remaining in the particles. This calculation was made assuming that all mass adsorbed by the particles before the sweep was CT. At the completion of the release experiments, the cell temperature was raised to $80 \mathrm{C}$, and after 2 days only a $28 \%$ of the mass adsorbed was removed by this procedure, which indicates that the remaining mass might be something other than CT. The long-term experiment was repeated, and again the MB rates agreed well with those calculated by GC, and with the prior experiments. After $209 \mathrm{~h}$ the CT desorption rate was 8.66x10-7 g CT/(g sample.h) and the mass adsorbed $0.00084 \mathrm{~g} / \mathrm{g}$ sample. After the test, the mesoporous silica was removed from the microbalance cell and an extraction was performed to determine the amount of CT left in the particles. Decane was used as solvent and the results indicate that only $8 \%$ of the remaining mass adsorbed in the particles was CT. This information was used to correct the actual CT mass adsorbed. Release rates were recalculated and plotted against the desorption rates obtaining, in a log-log plot, a curve that shows strong linearity of CT desorption rates with CT mass adsorbed over many orders of magnitude. Further experiments are underway with 40 and 60 Angstrom mesoporous silica particles to better characterize the effects of pore size distribution on CT release rates and long term sequestration. 